Infancia vulnerable y condiciones de acceso a la educación en el sector rural: el caso de la parroquia San José de Poaló, de la provincia de Cotopaxi

Número Publicado el 05 de julio de 2018

DOI: $10.23857 /$ dc.v4i3.795

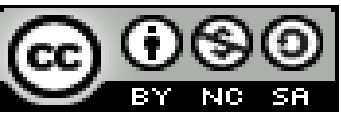

Ciencias de la educación

Artículo de investigación

\title{
Infancia vulnerable y condiciones de acceso a la educación en el sector rural: el caso de la parroquia San José de Poaló, de la provincia de Cotopaxi
}

\section{Vulnerable childhood and conditions of access to education in the rural sector: the case of the San José de Poaló parish, in the province of Cotopaxi}

\section{Infância vulnerável e condições de acesso à educação no setor rural: o caso da paróquia de San José de Poaló, na província de Cotopaxi}

\author{
Lourdes Y. Cabrera-Martínez ${ }^{\mathrm{I}}$ \\ lourdes.cabrera@utc.edu.ec \\ Wilmer R. Lozada-Guanotuña II \\ wilmer.lozada0@utc.edu.ec \\ Marcos E. Pacheco-Díaz III \\ erkmark95@gmail.com \\ Ricardo F. Ureña-López ${ }^{\text {IV }}$ \\ ricardo.urena@utc.edu.ec
}

Recibido: 25 de enero de 2018 * Corregido: 10 de marzo de $2018 *$ Aceptado: 28 de mayo de 2018

I. Magister en Ciencias Sociales con mención en sociología. Licenciada en Comunicación Social. Docente de la carrera de Comunicación Social de la Universidad Técnica de Cotopaxi, Latacunga, Ecuador.

II. Egresado de la carrera de Comunicación Social de la Universidad Técnica de Cotopaxi, Latacunga, Ecuador.

III. Egresado de la carrera de Comunicación Social de la Universidad Técnica de Cotopaxi, Latacunga, Ecuador.

vv. Magister en Ciencias Sociales con mención en sociología. Docente de la Carrera de Comunicación Social de la Universidad Técnica de Cotopaxi, Latacunga, Ecuador. 
Infancia vulnerable y condiciones de acceso a la educación en el sector rural: el caso de la parroquia San José de Poaló, de la provincia de Cotopaxi

\section{Resumen}

El análisis de las condiciones de acceso a la educación es una tarea que requiere de una evaluación permanente, en el caso de Ecuador se han presentado varias reformas en este campo y por ello se consideró importante examinar los cambios que se han generado, sobretodo, en el sector rural, en donde se presentan mayores dificultades respecto a tasas de escolarización, salud, alimentación entre otros. En ese sentido, el objetivo del presente artículo es exponer los resultados de un proyecto de investigación que analizó en qué condiciones los niños y niñas de 6 a 11 años de la parroquia San José de Poaló, ubicada en el cantón Latacunga, acceden al sistema educativo. Se usó el enfoque cualitativo a través del método etnográfico que estuvo integrado por dos elementos: la observación participante y la entrevista no directiva; el instrumento usado fue un temario de preguntas, el cual permitió guiar este estudio. Entre los resultados más importantes se destacan el valor asignado por parte de los niños y niñas, así como sus familias a la educación; adicionalmente se evaluaron las condiciones de transporte, salud, alimentación y entorno. Se plantea como conclusiones la necesidad de generar políticas públicas acordes a las dinámicas de los contextos, reconociendo sus características sociales, económicas y culturales. De igual manera es fundamental evaluar permanentemente los requerimientos que se presentan en el campo educativo en las parroquias rurales de Ecuador.

Palabras clave: Ecuador; educación; infancia; Latacunga; vulnerabilidad.

\section{Abstract}

The analysis of the conditions of access to education is a task that requires a permanent evaluation, in the case of Ecuador, several reforms have been presented in this field and with regard to the changes that have been generated, especially in the rural sector, where there are greater difficulties than school enrollment, health, food, among others. In this sense, the objective of this article is to present the results of a research project that analyzed the conditions in which children from 6 to 11 years of age from the San José de Poalo parish, in the canton of Latacunga, access the educational system. The qualitative approach was used through the entographic method that consisted of two elements: participant observation and non-directive interview; the instrument used was a set of questions, which allowed us to guide this study. Among the most important results are the value assigned by children and their families to education; additionally, transport, health, food and environment conditions were evaluated. The conclusions are the need to generate public policies according to the dynamics of the contexts, recognizing their social, economic and cultural characteristics. In the same way it is 
Infancia vulnerable y condiciones de acceso a la educación en el sector rural: el caso de la parroquia San José de Poaló, de la provincia de Cotopaxi

fundamental to permanently evaluate the requirements that are presented in the educational field in the rural parishes of Ecuador.

Keywords: Ecuador; education; childhood; Latacunga; vulnerability.

\section{Resumo}

A análise das condições de acesso à educação é uma tarefa que requer avaliação contínua, no caso do Equador tem havido várias reformas neste campo e foi considerado importante para examinar as mudanças que geraram, especialmente em o setor rural, onde há maiores dificuldades com relação às taxas de matrícula, saúde, nutrição, entre outras. Nesse sentido, o objetivo deste artigo é apresentar os resultados de um projeto de pesquisa que analisou as condições em que crianças de 6 a 11 anos da paróquia San José de Poalo, localizada no cantão Latacunga, o acesso à educação. A abordagem qualitativa foi utilizada por meio do método etnográfico, composto por dois elementos: observação participante e entrevista não diretiva; o instrumento utilizado foi um conjunto de perguntas, o que nos permitiu orientar este estudo. Entre os resultados mais importantes estão o valor atribuído pelas crianças e suas famílias à educação; além disso, as condições de transporte, saúde, alimentação e meio ambiente foram avaliadas. As conclusões são a necessidade de gerar políticas públicas de acordo com a dinâmica dos contextos, reconhecendo suas características sociais, econômicas e culturais. Da mesma forma, é fundamental avaliar permanentemente os requisitos que são apresentados no campo educacional nas paróquias rurais do Equador.

Palavras chave: Equador; educação; infância; Latacunga; vulnerabilidade.

\section{Introducción}

En Ecuador existe una deuda histórica con el desarrollo de la niñez, si bien se ha intentado implementar políticas que garanticen el adecuado cumplimiento de sus derechos, aún es necesario realizar acciones integrales que permitan el crecimiento pleno de niños y niñas. En el "Estado de los Derechos de la Niñez y la Adolescencia en Ecuador 1990 -2011" realizado por el Observatorio de los Derechos de la Niñez y la Adolescencia (ODNA, 2011) se afirma que el Movimiento de la Infancia nace en la década de los años 80 y se consolida en 1990 con la ratificación de la Convención de los Derechos del Niño.

El pleno reconocimiento de los Derechos de la Niñez, de acuerdo a ODNA, se produce con la creación del Código de la Niñez, documento que incorpora elementos de la constitución de 1998, las reformas 
Infancia vulnerable y condiciones de acceso a la educación en el sector rural: el caso de la parroquia San José de Poaló, de la provincia de Cotopaxi

al Código de Menores (1992) y el Foro de la Niñez (1990). En esta breve línea histórica se reflejan los avances y esfuerzos, al menos en materia legal, por mejorar la situación de indefensión a la que ha estado expuesta la niñez en el país. Sin embargo, si examinamos las condiciones estructurales en las que se desenvuelven, el escenario es poco favorable.

En Ecuador, según el censo realizado en el año 2010 por el Instituto Nacional de Estadísticas y Censos (INEC), existen cerca de 6 millones de niños, niñas y adolescentes. Dentro de este grupo humano hay que hacer una diferenciación en cuanto a la mejora en sus condiciones de vida, durante los últimos años. La Secretaría Nacional de Planificación y Desarrollo (SENPLADES), en el Plan Nacional del Buen Vivir (PNVB, 2009) establecido para los años 2013 a 2017 señala:

La costa es la región con menos niños y niñas desnutridos del país. Sus cifran se asemejan a las del área urbana, con el 16\% de desnutrición infantil. Un gran esfuerzo se observa en la Amazonía donde se ha logrado reducir la desnutrición a menos del $30 \%$ en los últimos cuatro años. Finalmente, en la niñez de la Sierra, el descenso de la desnutrición es casi nulo en los últimos seis años. Allí habita un alto porcentaje de niños y niñas indígenas que, desde las primeras mediciones de la desnutrición son los más afectados de todo el país. (p.162)

Cotopaxi es una provincia ubicada en la región sierra-centro del país y de acuerdo a un estudio realizado por ODNA, sobre el índice de cumplimiento de derechos de niños, niñas y adolescentes esta provincia ocupa el puesto 22 entre las 22 analizadas, con una calificación de 1,8 sobre 10 puntos, es decir, no existe garantía para el desarrollo pleno de los niños y niñas. Otro de los problemas a los que se enfrenta la niñez en la serranía ecuatoriana es el relacionado con los índices de escolaridad, la sierra es el sector más rezagado con respecto al año 2001 (SENPLADES, 2013) En este escenario, la población infantil es un grupo vulnerable en diversos ámbitos, en este trabajo se presentará de manera específica la dimensión educativa de la problemática.

En este escenario, otro aspecto a considerar es que en Ecuador desde el año 2010 se implementó un nuevo modelo de gestión educativa que, según las autoridades de educación, buscaba descentralizar el sistema a través de la creación de zonas, distritos y circuitos. Las zonas son encargadas de la planificación, coordinación, gestión y control, a nivel macro; los distritos de la coordinación, microplanificación, gestión y control en el territorio; en el caso de los circuitos educativos su función 
Infancia vulnerable y condiciones de acceso a la educación en el sector rural: el caso de la parroquia San José de Poaló, de la provincia de Cotopaxi

es la oferta educativa para los niveles Inicial, General Básica, Bachillerato y Educación para Adultos. Este nuevo modelo implicó el cierre de varias escuelas unidocentes, ubicadas en el sector rural.

El referente teórico utilizado para este trabajo es vulnerabilidad, enfoque asociado a dos grandes líneas, por un lado, la idea de fragilidad/precariedad; y, por otro lado, riesgo. Los principales debates alrededor de vulnerabilidad hacen énfasis en la necesidad de comprender a la vulnerabilidad más allá y no solo asociada a la idea de pobreza (Golovanevsky, 2007; Infante et. al. 2012).

El objetivo es ampliar la comprensión de un fenómeno complejo, de este modo se contribuye a un "análisis multidimensional, que atiende no solo a lo económico, como puede hacer la pobreza (al menos en una visión clásica) ...” (Infante et. al. 2012).

Caroline Moser en 1998 plantea el denominado Asset vulnerability framework y "a partir de numerosos trabajos empíricos realizados para el Banco Mundial sobre familias pobres en distintos países del mundo, la autora propone prestar mayor atención a lo que los pobres poseen más que a lo que carecen” (González, 2009, p.3). Más adelante, en 1999 serán Rubén Eastman y Carlos Filgueira, quienes retoman esta propuesta de Moser y realizan aportes en función de la realidad de América Latina. En "Vulnerabilidad, Activos y Exclusión Social en Argentina y Uruguay" un trabajo realizado para la Organización Internacional del Trabajo (OIT), señalan la importancia de "el énfasis puesto en la familia y sus recursos como eje explicativo de los fenómenos dinámicos de reproducción del bienestar de los hogares; pero advierten una fuerte influencia de paradigmas de orientación liberal" (González, 2009, p.4).

A partir del año 2000 los análisis estarán marcados por ese debate, entre los estudios que aparecen constan: "Estructura de oportunidades y vulnerabilidad social, aproximaciones conceptuales recientes" (Carlos Filgueira, 2001); "Seducidos y abandonados: pobres urbanos, aislamiento social y políticas públicas" "Notas sobre la medición de la vulnerabilidad social" (Kaztman, 2000). Estas investigaciones realizadas principalmente para la Comisión Económica para América Latina y el Caribe (CEPAL) y la Universidad de Uruguay coinciden en analizar la vulnerabilidad en términos de exclusión y lo hacen en función de las dimensiones de educación, trabajo y residencia. Tal es el caso de trabajos como "Pobreza, exclusión y vulnerabilidad social" (Busso, 2005), y "Vulnerabilidad social: una propuesta para su medición en Argentina" (Golovanevsky, 2007) ambos autores coinciden en analizarla desde el enfoque AVEO, propuesto por Kaztman y Filgueira (1999), y proponen tomar 
Infancia vulnerable y condiciones de acceso a la educación en el sector rural: el caso de la parroquia San José de Poaló, de la provincia de Cotopaxi

en cuenta tanto los activos que poseen las familias, así como las oportunidades que provienen del Estado, el mercado y la sociedad.

\section{Para Nanculeo y Merino (2016) el enfoque de la vulnerabilidad está centrado en tres factores:}

“1) un bajo nivel de activos económicos en relación con las necesidades del hogar, 2) un nivel de exposición a riesgos económicos que es elevado en relación con los activos del hogar, 3) una ausencia relativa de mecanismos de defensa frente a riesgos" (p. 54-55).

Estos aspectos inciden de manera diferente según las condiciones de vulnerabilidad social que viven las familias. Otro elemento que se destaca en estas aproximaciones es el papel que desempeña la constitución de las familias, en un análisis realizado a zonas vulnerables en Uruguay se determinó los desafíos que se les presentaron a las familias a partir de mediados del siglo XX, y se determinó que sus estructuras quedaron debilitadas por dos razones: (1) transformaciones sociales, e (2) Inercia estatal. El objetivo del estudio fue explicar cómo la estructura familiar incide en el rendimiento educativo de los niños y niñas en los estratos bajos urbanos (Kaztman y Rodríguez, 2006).

Sumado a estos elementos, un aspecto sobre el que se reflexiona en la problemática sobre vulnerabilidad es el referido a la constitución de los vínculos que pueden ser fuente de capital social. De León-Torres (2014), le otorga especial importancia y propone que es preciso reflexionar sobre el debilitamiento de relaciones de reciprocidad que, en décadas pasadas, fueron un soporte importante para las familias y por tanto para el cuidado de niños y niñas.

En este contexto, el presente artículo expone los resultados del trabajo investigativo "Vulnerabilidad educativa en la población infantil” realizado en la parroquia San José de Poaló, ubicada en la parte occidental del cantón Latacunga. Como se mencionó anteriormente, la provincia de Cotopaxi presenta una serie de características que vulneran, de manera prioritaria, los derechos de la infancia. En ese sentido, la problemática identificada está asociada a las condiciones en las que acceden a la educación niños y niñas, en edades comprendidas entre 5 y 11 años. En función de esto la pregunta que guio la investigación fue ¿De qué manera perciben el acceso a la educación básica los infantes de la parroquia San José de Poaló? Tal como lo sugieren los enfoques sobre vulnerabilidad, esta debe ser comprendida en sus dimensiones objetivas y subjetivas, es decir, se debe buscar conocer los recursos materiales 
Infancia vulnerable y condiciones de acceso a la educación en el sector rural: el caso de la parroquia San José de Poaló, de la provincia de Cotopaxi

con los que cuentan, así como la percepción de este escenario. Para ello fue importante trabajar de manera cercana con las familias y los infantes.

El objetivo propuesto fue determinar cómo conciben las condiciones de acceso al sistema educativo las familias y los infantes de la parroquia San José de Poaló, es decir, conocer las características de la educación en un contexto rural. Para cumplir con esta finalidad se plantearon como objetivos específicos: a) Identificar el sentido que los niños y niñas le asignan al acceso a la educación; b) Examinar cómo perciben las condiciones de vialidad de la parroquia; y c) Explicitar la calidad en el servicio de transporte al interior de la parroquia.

Este trabajo se realizó debido al permanente interés de la Universidad Técnica de Cotopaxi por vincularse con los sectores vulnerables. El aporte de esta iniciativa es brindar información que permita conocer las dificultades, así como las estrategias que generan los infantes y sus familias para acceder al sistema educativo. Se aspira a sensibilizar a la sociedad, así como a las autoridades e instituciones, de tal manera que se pueda generar políticas públicas dirigidas a mejorar la calidad de vida de niños y niñas, quienes tienen limitantes a la hora de acceder a su derecho a la educación: nutrición, transporte, aulas cómodas y espacios de entretenimiento.

\section{Materiales y Métodos}

En la investigación se utilizó un enfoque cualitativo, definido por LeCompte (1995) como "una categoría de diseños de investigación que extrae descripciones a partir de observaciones que adoptan la forma de entrevistas, narraciones, notas de campo, grabaciones, transcripciones de audio y video casette, registros escritos de todo tipo, fotografías o películas y artefactos" (p.7).

Se trabajó mediante el método etnográfico, que permitió acompañar a diferentes familias y de esta manera conocer de cerca cómo acceden al sistema educativo. Para el caso de este trabajo se obtuvo información a través de entrevistas, grabaciones y fotografías que ayudaron al desarrollo del trabajo de campo con la participación de familias y niños/as de diferentes barrios de la parroquia San José de Poaló.

El nivel de investigación en el proyecto fue exploratorio-descriptivo, debido a que se buscó sondear problemas poco analizados. En primer lugar, se acudió a la parroquia de Poaló para detectar informantes clave y caracterizar el escenario, de esta manera se pudo conocer de mejor manera el tema 
Infancia vulnerable y condiciones de acceso a la educación en el sector rural: el caso de la parroquia San José de Poaló, de la provincia de Cotopaxi

a estudiar, es decir, permitió recoger elementos para aclarar la problemática a estudiar, así como los objetivos.

\section{Población y Muestra}

La población que participó de este estudio fue la parroquia San José de Poaló, del cantón Latacunga, específicamente aquellos niños en edad escolar (6 a 11 años) y sus familias. En el proyecto se utilizó un muestreo no probabilístico de tipo intencional. Los criterios que se determinaron en el equipo de investigación para seleccionar a los sujetos de estudio que integraron la muestra fueron:

Familias e infantes que provengan de diferentes barrios de la parroquia.

Enfoque de género

\section{Técnicas e instrumentos}

El uso del método etnográfico se llevó a cabo a través de las técnicas de la observación participante y entrevista etnográfica. En el caso de la observación participante se la comprende, como señala Ginzburg: "Ver para entender: afinar la vista, el oído, la sensibilidad". Lo que se busca es lograr el contacto directo con la vida cotidiana de la población, de este modo se garantiza la confiabilidad de la información recopilada y el aprendizaje de los sentidos implícitos a dichas prácticas (Guber, 2015). La implementación de la etnografía implicó un acompañamiento durante cuatro meses a seis familias, quienes permitieron al equipo investigador ser parte de su día a día, de este modo se pudo observar en su propio contexto las condiciones, así como la percepción de los niños y las familias de la parroquia de Poaló frente a una dinámica educativa.

\section{Instrumento para Observación sistemática}

\section{Diario de campo}

\section{Entrevista etnográfica:}

Es una estrategia muy útil que permite hacer que la gente hable sobre lo que conoce, piensa y cree. En el proceso de la investigación la entrevista involucra dos momentos (Guber, 2015).

Apertura: el investigador debe encontrar las preguntas más relevantes tomando en cuenta la perspectiva del informante.

Focalización y profundización: implementación de preguntas relevantes que permitan la ampliación y sistematización de lo más importante. 
Infancia vulnerable y condiciones de acceso a la educación en el sector rural: el caso de la parroquia San José de Poaló, de la provincia de Cotopaxi

\section{Resultados y discusión}

Uno de los resultados más significativos que arrojó esta investigación fue el valor que tiene la educación para los niños y niñas que participaron de este estudio, aunque esto no implica la exclusión de problemas en el ambiente escolar. Pedro (nombre ficticio) un niño de 10 años aseguró:

Mis profesores me tratan bien, me enseñan y me ayudan cuando no entiendo algo, de mi escuela me gustan los minutos cívicos y se refieren y exponen a las fechas más importantes del Ecuador. Lo que no me gustan de mi institución es que los profesores les dejan a algunos estudiantes a supletorio o remedial y para ayudarles piden dinero u algo. Nunca me he peleado en la escuela. Es importante ir a una institución educativa para prepararse y llegar a tener algo y ser alguien en la vida (Pedro, comunicación personal).

En coincidencia con esta experiencia, Fernando, un niño de 11 años expresó:

Mis profesores me tratan con mucho cariño y respeto. Lo que más me gusta de mi escuela es las paredes, los árboles, las plantas y las aves. Mi desayuno escolar es una colada y lo recibo a las 9:30 y me gusta. He tenido problemas en la escuela con mis compañeros porque me quitaban las cosas y no me devolvían. Es importante ir a la escuela para estudiar, aprender y ser bueno en el futuro (Fernando, Comunicación personal).

Otro de los niños, Rafael, un niño de 11 años, quien vive con su padre y madre, revela su preocupación por los actos violentos que se generan en la escuela, un espacio que para él es fundamental:

De grande me gustaría ser científico porque me gusta la ciencia e investigar sobre la vida antigua y el futuro y lo voy a conseguir estudiando bastante siendo un ejemplo. En la escuela me tratan bien, solo nos hablan cuando no hacemos las tareas. De mi escuela me gustan los espacios y mis amigos. De mi escuela no me gustan los jóvenes que maltratan a los más pequeños y nos hacen bullying. En la escuela me hacían bullying y me rompieron un trabajo. Es importante ir a la escuela para estudiar y no andar en malos pasos y es bueno porque es un espacio de enseñanza (Rafael, comunicación personal).

Precisamente estos aspectos que fueron relatados por los niños y niñas son coincidentes con lo planteado respecto a los múltiples factores que intervienen en la vulnerabilidad:

...la condición dinámica que resulta de la interacción de una multiplicidad de factores de riesgo y protectores individuales y contextuales (familia, escuela, barrio, comuna) antes y durante el desarrollo educativo de un niño o niña, que se manifiestan en conductas o hechos de mayor o menor riesgo social, 
Infancia vulnerable y condiciones de acceso a la educación en el sector rural: el caso de la parroquia San José de Poaló, de la provincia de Cotopaxi

económico, psicológico, cultural, ambiental y/o biológico, produciendo una desventaja comparativa entre sujetos, familias y/o comunidades ( Junaeb, 2005, pp. 48, citado en Ñanculeo y Merino, 2016). Respecto de sus aspiraciones a futuro, los niños y niñas entrevistados expresaron como opciones profesionales el ser pintores, profesores, científicos, futbolistas, mecánicos y policías. Todos coinciden en que para lograrlo se requiere mucho esfuerzo y compromiso de su parte.

Otro de los elementos presentes en la conversación con los niños y niñas fue la percepción que tienen de su barrio. Para los infantes su barrio es muy importante, además valoran las fiestas de su comunidad, la naturaleza, contar con amigos y familiares; sin embargo, una de las cosas que les desagradan son la inseguridad, así como la falta de servicios básicos: "Lo que me gusta de mi barrio son los animales, las personas y mis amigos, lo que no me gusta es la inseguridad en las calles y la falta de recursos como lámparas, ensanchamiento de las calles y alcantarillado" (Rafael, comunicación personal).

Además, el equipo investigador entrevistó a los padres de estos niños/as y coincidieron en que un elemento a tomar en cuenta para el análisis de la realidad educativa de esta parroquia es que el modelo de gestión educativa en algunos les significó beneficios en sus trámites porque ya no tienen que trasladarse a otros lugares para hacerlo; sin embargo, también expresaron su preocupación, para varias familias esta situación les perjudicó porque sus hijos tienen que trasladarse largas distancias. La observación y conversación con los padres y madres de familia permitió detectar los roles que estos actores cumplen en los escenarios educativos, así como en la forma de enfrentar distintas situaciones de vulnerabilidad, es así que se generan nuevas demandas por parte de las familias. Así lo expresan Rodríguez y Puertas (2016):

Las familias han ido evolucionando, desarrollando un criterio más profundo y fundamentado de lo que consideran educativamente adecuado para sus hijos e hijas, y en consecuencia han ido volcando expectativas más precisas y elaboradas que exigen respuestas e interacciones también más elaboradas por parte de los profesionales. (p. 59)

Uno de los aspectos detectados fue la serie de problemas que se presentaron para el traslado a las diferentes escuelas debido a que han tenido que hacerlo caminando, el tiempo de traslado oscila entre 15 minutos (en unos casos) a una hora. A esto suma el servicio de transporte al interior de los barrios de la parroquia: en el caso de los buses no circulan de manera permanente y por ello muchas de las 
Infancia vulnerable y condiciones de acceso a la educación en el sector rural: el caso de la parroquia San José de Poaló, de la provincia de Cotopaxi

veces deben tomar camionetas, exponiéndose a una serie de peligros. Sobre el transcurso de ida y vuelta desde su casa a las instituciones escolares, algunos moradores de la parroquia expresaron que "los infantes están expuestos a peligros como: secuestros o manipulaciones por personas que no pertenecen a la parroquia".

A esto hay que agregarle los problemas de salud presentes en los barrios de la parroquia, y que afectan la calidad de vida de los infantes, así como su desempeño académico. Susana Baéz, Directora del Centro de Salud de Poaló expresó que "las principales enfermedades que padecen los niños y niñas de entre 6 a 11 años, son las infecciones respiratorias generadas por el polvo de las calles o el frío característico del lugar".

La alimentación es un elemento esencial para que los niños/as puedan conseguir un rendimiento óptimo en sus escuelas, de las entrevistas realizadas, así como de la observación se pudo detectar que en ocasiones los infantes no integran en su dieta alimentos de todos los grupos nutricionales, muchas veces lo hacen por acudir rápido a las escuelas; es usual que el desayuno esté acompañado de algunos de estos alimentos: arroz, papas, pan, huevos y café. Además, los niños comentaron que reciben el desayuno escolar, el cual consiste en una colada acompañada de una galleta cereal, sin embargo, esto no garantiza la suficiente nutrición que requiere el niño o niña para desarrollar su aprendizaje.

Tal como se acaba de exponer, la vulnerabilidad está asociada a una serie de factores asociados al contexto sociocultural, familiar e individual. Sobre ello Ñanculeo y Merino (2016) afirman:

Los factores protectores y de riesgo que determinan el nivel de vulnerabilidad, para el caso del desarrollo infantil, se encuentran en distintos planos, desde lo individual a lo contextual, abarcando diferentes dimensiones tales como el desarrollo psicoemocional, la salud, la estructura y dinámica familiares, el contexto socioeconómico de la familia, el escolar y el barrial o comunitario, entre otros (p.60).

Un aspecto que debe destacarse es que pese a las diversas condicionantes que, en muchos de los casos, limitan una educación integral los padres y madres de los infantes asignan un valor importante a la educación y en ninguno de los casos se plantearon la posibilidad de retirar al niño del sistema educativo, sino que consideraban a la educación como un recurso importante para garantizar a sus hijos mejores condiciones de vida en lo personal y profesional. 
Infancia vulnerable y condiciones de acceso a la educación en el sector rural: el caso de la parroquia San José de Poaló, de la provincia de Cotopaxi

\section{Conclusiones}

El acceso a la educación en el sector rural ecuatoriano requiere de cambios urgentes, porque tal como se acaba de exponer en el caso de esta parroquia rural, ubicada en el cantón Latacunga, se pudo evidenciar diferentes dificultades que deben enfrentar niños y niñas de entre 6 a 11 años para acceder a su derecho a la educación.

Los niños y niñas que participaron en este estudio asignan un valor importante a la educación, pese a que tienen que enfrentar limitantes en salud, vivienda, alimentación, entre otros. Un elemento convergente en los testimonios de estos infantes fue la prospectiva que realizan respecto de sus estudios, es decir, se planteaban un escenario a futuro en donde la educación es una dimensión trascendental para su formación. En el caso de los niños hubo una mayor inclinación por la carrera policial, futbolistas y mecánicos; y en el caso de las niñas se dio una mayor preferencia por profesiones como maestra y enfermera, es decir, hay una elección asociada a roles de género asignados socialmente a mujeres y hombres.

Uno de los elementos que deja planteado esta investigación es la necesidad de que los estados elaboren políticas públicas tomando en cuenta las condiciones y requerimientos de los propios actores involucrados, en este caso de los niños/as, así como de las familias que habitan en la parroquia San José de Poaló. Además, es importante reflexionar que para que una política funcione hay que diseñarla e implementarla de manera integral, caso contrario tendrá serias dificultades en su aplicación. En este caso se diseñó un nuevo modelo de gestión educativa, pero debido a los problemas en salud, vivienda y transporte se dificulta la posibilidad de hablar de un modelo educativo exitoso.

\section{Referencias Bibliográficas}

Busso, G. (2005). Pobreza, exclusión y vulnerabilidad social. Usos, limitaciones y potencialidades para el diseño de políticas de desarrollo y de población"; Tandil, VIII Jornadas Argentinas de Estudios de Población, Asociación de Estudios de la Población Argentina.

De León-Torres, M. S. (2014). Niños, niñas, y mujeres: Una amalgama vulnerable. Revista Latinoamericana de Ciencias Sociales, Niñez y Juventud, 12 (1), 105-119.

Filgueira, C. (2001). Estructura de oportunidades y vulnerabilidad social: aproximaciones conceptuales recientes. Seminario internacional "Las diferentes expresiones de la vulnerabilidad 
Infancia vulnerable y condiciones de acceso a la educación en el sector rural: el caso de la parroquia San José de Poaló, de la provincia de Cotopaxi

social en América Latina y el Caribe”. Comisión Económica para América Latina y el Caribe. Santiago de Chile.

Golovanevsky, L. (2007) Vulnerabilidad Social: una propuesta para su Medición en Argentina. Revista de Economía y Estadística - Volumen. XLV - Nº 2 - (2007), 53-94

González, L.(2009). Orientaciones de lectura sobre Vulnerabilidad Social. En L. González (Comp.) Lecturas sobre vulnerabilidad y desigualdad social (pp. 1 - 14). Córdoba: Centro de Estudios Avanzados - CONICET.

Guber, R. (2015). La etnografía. Método, campo y reflexividad. México: Editorial Siglo XXI Editores. Infante, M., Matus, C., Paulsen, A., Salazar, A., Vizcarra, R. (2012). Narrando la vulnerabilidad escolar: performatividad, espacio y territorio. Chile: Literatura y lingüística $\mathrm{N}^{0} 7$.

Kaztman, R. (2000). Notas sobre la medición de la vulnerabilidad social. Buenos Aires: Comisión Económica para América Latina.

Kaztman, R., y Rodríguez, F. (2006). Las formas de constitución de las familias pobres urbanas en Uruguay: consecuencias sobre el rendimiento educativo de los niños. Montevideo, Prisma 21.

LeCompte, M. D. (1995). Un matrimonio conveniente: Diseño de investigación cualitativa y estándares para la evaluación de programas. Revista Electrónica de Investigación y Evaluación Educativa. 1 (1), 1-13.

Ñanculeo, R. Y Merino, J. (2016). Una aproximación a la vulnerabilidad en el sistema de educación parvularia en Chile. Noesis. Revista de Ciencias Sociales y Humanidades, 25 (50), 51-88.

Observatorio de los Derechos de la Niñez y la Adolescencia. (2011). Los derechos de la niñez y la adolescencia en Cotopaxi. Ecuador.

Rodríguez, A. y Puertas, M. (2016). El valor de lo público en educación infantil. Revista Interuniversitaria de Formación del Profesorado, 30 (1), 57-66.

SENPLADES (2009) Plan Nacional para el Buen Vivir 2013-2017, Todo el mundo mejor. Quito: Secretaría Nacional de Planificación y Desarrollo. 